\title{
COOPETICIÓN EN LA GESTIÓN ACADÉMICA DE LA EDUCACIÓN SUPERIOR EN RIO GRANDE DO SUL, BRASIL
}

\section{COOPETITION IN THE ACADEMIC MANAGEMENT OF HIGHER EDUCATION IN RIO GRANDE DO SUL, BRAZIL}

\author{
Hildegard Susana JUNG ${ }^{1}$ \\ Louise de Quadros DA SILVA² \\ Douglas VAZ ${ }^{3}$ \\ Paulo FOSSATTI ${ }^{4}$
}

\begin{tabular}{|lll|}
\hline Recibido & $:$ & 28.01 .2021 \\
Aceptado & $:$ & 13.06 .2021 \\
Publicado & $:$ & 02.08 .2021 \\
\hline
\end{tabular}

\begin{abstract}
RESUMEN: Especialmente en tiempos de crisis, como la pandemia del coronavirus, la cooperación se convierte en una estrategia fundamental para la viabilidad institucional. Se trabaja con el objetivo de sistematizar las experiencias de coopetición entre las Instituciones de Educación Superior del Consorcio de Universidades Comunitarias del Estado de Rio Grande do Sul, Brasil (COMUNG) a través de la participación en actividades que promuevan la gestión sostenible. Se trata de un estudio de caso, de carácter cualitativo, para el cual se realizó una revisión de la literatura y se preparó un diario de campo con base en las observaciones y experiencias de los autores, también como gestores de una universidad que forma parte de COMUNG. Entre los principales resultados destacamos la coopetición como: a) estrategia de fortalecimiento de instituciones educativas en tiempos de crisis con agencias gubernamentales; b) Fortalecimiento del espacio entre universidades para la gestión sostenible. Concluimos por la coopetición como una estrategia asertiva y potencializadora del empoderamiento del COMUNG para enfrentar sus desafíos, especialmente en tiempos de COVID-19.
\end{abstract}

Palabras clave: Coopetición; Gestión Universitaria; COMUNG, Brasil, Coronavirus.

ABSTRACT: Especially in times of crisis, such as the coronavirus pandemic, cooperation becomes a fundamental strategy for institutional viability. We work with the objective of systematizing the experiences of coopetition of Higher Education Intitutions of the Consortium of Community Universities of the State of Rio Grande do Sul (COMUNG) through participation in activities that promote sustainable management. This is a qualitative case study, for which we conducted a literature review and prepared a field diary based on the observations and experiences of the authors, as well as managers of a university belonging to COMUNG. Among the main results, we highlight the coopetition as: a) strategy to strenghthen educational institutions in times of crisis with government agencies; b) strenghthening space among universities for sustainable management. We conclude that coopetition is an assertive and empowering strategy for COMUNG to face its challenges in times of COVID-19.

Keywords: Coopetition; University Management; COMUNG, Brazil, Coronavirus.

\footnotetext{
${ }^{1}$ Doctora en Educación. Docente del Curso de Pedagogía, profesora permanente y coordinadora del Programa de Posgrado (PPG) en Educación de la Universidad La Salle Canoas. Brasil. E-mail: hildegard.jung@unilasalle.edu.br. ORCID: https://orcid.org/0000-0001-5871-3060

${ }^{2}$ Magíster en Educación por la Universidad La Salle Canoas. Integrante del grupo de investigación Gestão Educacional nos diferentes contextos. Brasil. E-mail: louise.quadrosdasilva@gmail.com. ORCID: https://orcid.org/0000-0002-8632-3374

${ }^{3}$ Estudiante del Doctorado en Educación en la Universidad La Salle Canoas. Integrante del grupo de investigación Gestão Educacional nos diferentes contextos. Brasil. E-mail: douglas.vaz@ unilasalle.edu.br. ORCID: https://orcid.org/0000-00023950-0120

${ }^{4}$ Doctor en Educación. Rector de la Universidad La Salle, Canoas/RS y docente del PPG en Educación de esta universidad. Líder del grupo de investigación Gestão Educacional nos diferentes contextos. Brasil. E-mail: paulo.fossatti@unilasalle.edu.br. ORCID: https://orcid.org/0000-0002-9767-5674
} 


\section{Journal of the Academy $|79|$}

\section{INTRODUCCIÓN}

La inesperada pandemia causada por el coronavirus está provocando importantes alteraciones en las formas de existencia y gestión. Cambia profundamente las relaciones sociales, económicas, culturales y educativas, a nivel local y global. En enero de 2020, el COVID-19 fue considerado una epidemia y, el 11 de marzo del mismo año, fue definido por la Organización Mundial de la Salud como una pandemia. Siguiendo la dinámica global, Brasil asumió los protocolos de salud proclamados por la Organización Mundial de la Salud (OMS), que están afectando directamente la gestión de las Instituciones de Educación Superior (IES).

Por ello, al igual que otros segmentos, en el ámbito educativo estamos constantemente comprometidos en reorganizar las estrategias de gestión para enfrentar los nuevos desafíos de sustentabilidad que trae la pandemia. Entre los diversos escenarios brasileños, las instituciones educativas pertenecientes al Consorcio de Universidades Comunitarias de Rio Grande do Sul (COMUNG) optan por la coopetición como estrategia de empoderamiento ante las amenazas externas e internas. Ella puede ser comprendida desde la perspectiva de Dal-Soto y Monticelli (2017), como un movimiento sinérgico que abarca la competencia y, al mismo tiempo, la cooperación entre instituciones.

En un plazo de apenas dos meses, considerando el recorte del 16 de marzo al 16 de mayo de 2020 para el presente artículo, se presentan acciones que orientan un movimiento colectivo con rapidez y toma de decisiones en un tiempo récord, nunca vivido en la institución. De carácter comunitario, confesional o no, sin fines de lucro, en su mayoría filantrópicos, estas IES son reconocidas por atender las demandas de sus comunidades dependiendo del pago de cuotas mensuales para su sostenibilidad económica y financiera. En el escenario de la pandemia, con el aislamiento social, esas instituciones han tenido que optar por la interrupción de las clases o la adopción de la educación remota, de manera síncrona. La segunda opción fue la elegida por el COMUNG.

Sin embargo, se pregunta cómo viabilizar y sensibilizar a los actores educativos y sociales a esta nueva forma de educar. Entre los innumerables intentos de respuesta, el COMUNG (2020a) opera con el concepto de coopetición como estrategia que oxigena su modelo de gestión académico e institucional aquí discutido. En este sentido, la motivación de nuestra investigación se debió a la reflexión sobre el momento actual, que demanda nuevos 


\section{Journal of the Academy $|80|$}

comportamientos de parte de las Instituciones de Educación Superior (IES). Además, la relevancia del estudio radica en la amplitud del tema de la coopetición en el sector educativo de la educación superior, que tiene mucho por explorar, tanto en el período pandémico como en el pospandémico, sea en el contexto de las IES del COMUNG, sea en lo que se refiere a otras alianzas.

A partir del contexto que se ha expuesto, el presente artículo tiene el objetivo de sistematizar las experiencias de coopetición entre las Instituciones de Educación Superior del Consorcio de Universidades Comunitarias del Estado de Rio Grande do Sul, Brasil (COMUNG) a través de la participación en actividades que promuevan la gestión sostenible. El problema que se buscó investigar está correlacionado al objetivo: ¿qué acciones han llevado a cabo las IES comunitarias del COMUNG para promover la gestión sostenible y que se pueden caracterizar como experiencias de coopetición?

Para dar respuesta a la pregunta anterior, la investigación presenta los conceptos de coopetición, además de ejemplos de actividades que se pueden caracterizar como coopetitivas, pues han auxiliado a las instituciones involucradas a enfrentar los retos de la contemporaneidad de forma conjunta. Además, esta perspectiva de coopetición entre las IES comunitarias del COMUNG todavía no ha sido objeto de investigación científica, por lo que se justifica una mirada atenta a esa realidad, pudiendo suscitar otros estudios en este campo. Se trata de un estudio de caso cualitativo, para el cual realizamos una revisión de la literatura y elaboramos un diario de campo basado en las observaciones y experiencias de los autores, como gestores de una universidad comunitaria en el sur de Brasil.

\section{DESARROLLO}

El marco teórico comienza conceptuando dos términos relevantes de la temática investigativa, que son la universidad comunitaria y el COMUNG. Según Quadros Da Silva (2019), se trata de instituciones con patrimonio de la sociedad civil o autoridades, que invierten íntegramente los recursos en sus actividades, desarrollando permanentemente acciones comunitarias. Así, dichas instituciones tienen derecho a participar en la asignación de recursos presupuestarios, así como en los avisos públicos reservados a las instituciones públicas. Según el art. 1 de la Ley 12.881, tienen características como personalidad jurídica, bienes públicos y no tienen finalidad de lucro (Brasilia, 2013). 


\section{Journal of the Academy $|81|$}

Las instituciones comunitarias a veces se confunden con instituciones públicas y privadas, ya que tienen características similares a ambas. Para una mejor comprensión de las diferencias y similitudes entre universidades públicas, privadas y comunitarias, se presenta a continuación la imagen de Schmidt (2018) en la Tabla 1.

Sin embargo, antes de profundizar la identidad de las Instituciones Comunitarias de Educación Superior (ICES), registramos que, según la legislación brasileña, tenemos nuestras propias identidades, según la categoría: pública, privada y comunitaria. Las privadas y comunitarias todavía pueden ser confesionales. Sin embargo, es importante colocar a las instituciones del COMUNG en la categoría de instituciones comunitarias, como se muestra a continuación.

\section{Tabla 1}

Características de las IES públicas, comunitarias y privadas

\begin{tabular}{|c|c|c|c|}
\hline & IES públicas & IES Comunitarias & Privadas (particulares) \\
\hline $\begin{array}{l}\text { Iniciativa de } \\
\text { creación }\end{array}$ & $\begin{array}{l}\text { Gobierno federal, } \\
\text { estadual o municipal }\end{array}$ & $\begin{array}{l}\text { Entidades y liderazgos de } \\
\text { la sociedad civil }\end{array}$ & $\begin{array}{l}\text { Grupo privado } \\
\text { (empresarial o familiar) }\end{array}$ \\
\hline $\begin{array}{l}\text { Responsabilida } \\
\text { d por el } \\
\text { mantenimiento }\end{array}$ & $\begin{array}{l}\text { Gobierno federal, } \\
\text { estados o municipios }\end{array}$ & $\begin{array}{l}\text { Entidades de la sociedad } \\
\text { civil y del poder público } \\
\text { local }\end{array}$ & $\begin{array}{l}\text { Grupo privado } \\
\text { (empresarial o familiar) }\end{array}$ \\
\hline $\begin{array}{l}\text { Naturaleza y } \\
\text { finalidad }\end{array}$ & Pública estatal & Pública no estatal & Privada \\
\hline $\begin{array}{l}\text { Destino de los } \\
\text { resultados } \\
\text { económicos }\end{array}$ & $\begin{array}{l}\text { Inversión en la propia } \\
\text { universidad }\end{array}$ & $\begin{array}{l}\text { Inversión en la propia } \\
\text { universidad }\end{array}$ & Apropiación privada \\
\hline $\begin{array}{l}\text { Forma de } \\
\text { elección } \\
\text { de los dirigentes }\end{array}$ & $\begin{array}{l}\text { En las Instituciones } \\
\text { Federales, } \\
\text { nombramiento por el } \\
\text { Presidente de la } \\
\text { República a partir de } \\
\text { una tríplice lista }\end{array}$ & $\begin{array}{l}\text { Elección (en algunas } \\
\text { instituciones) o } \\
\text { nombramiento por la } \\
\text { mantenedora (en } \\
\text { otras instituciones) }\end{array}$ & $\begin{array}{l}\text { Nombramiento por la } \\
\text { mantenedora }\end{array}$ \\
\hline
\end{tabular}




\section{Journal of the Academy $|82|$}

$\begin{array}{llll}\text { Forma de } & \text { Autarquía o fundación } & \text { Mantenida por asociación } & \text { Mantenida por una } \\ \text { constitución } & \text { pública } & \text { o fundación } & \begin{array}{l}\text { corporación (anónima o } \\ \end{array} \\ & & \text { limitada) }\end{array}$

Fuente: Resumido a partir de Schmidt (2018, p. 45).

Según Schafer y Spengler (2019) las instituciones comunitarias se refieren a instituciones privadas, pero son entidades no gubernamentales y sin fines de lucro, que brindan servicios de carácter público. De acuerdo con esos autores, al Sur del país tenemos universidades comunitarias, predominantemente, con el compromiso de contribuir al desarrollo de la comunidad en la que operan. Las comunitarias están representadas por la Asociación Brasileña de Universidades Comunitarias (ABRUC), y esta abarca a cuatro entidades regionales: el Consorcio de Universidades del Estado de Rio Grande do Sul (COMUNG), que representa las instituciones del estado de Rio Grande do Sul; la Asociación de Fundaciones Educativas de Santa Catarina (ACAFE), en el estado de Santa Catarina; la Asociación Nacional de Educación Católica de Brasil (ANEC), de instituciones católicas de todo el país; y la Asociación Brasileña de Instituciones Educativas Evangélicas (ABIEE), de instituciones evangélicas. (Schmidt, 2018).

De esa manera, en Rio Grande do Sul (RS) tenemos el COMUNG, que surgió en 1993, siendo oficialmente constituido el 27 de abril de 1996. Lo integran 14 IES, con más de ocho mil profesores, constituyéndose como el mayor sistema de educación superior en funcionamiento en Rio Grande do Sul (COMUNG, 2020a). Según Palmeiras y Grzybovski (2016), el COMUNG es el mayor sistema de educación de Rio Grande do Sul y, según su plataforma digital (COMUNG, 2020a), está compuesto de las siguientes instituciones: Universidade Franciscana (UFN); Universidade Feevale; Pontifícia Universidade Católica do Rio Grande do Sul (PUCRS); Universidade de Caxias do Sul (UCS); Universidade de Cruz Alta (Unicruz); Universidade Regional do Noroeste do Estado do Rio Grande do Sul (Unijuí); Universidade La Salle (Unilasalle); Universidade de Santa Cruz do Sul (Unisc); Universidade do Vale do Rio dos Sinos (Unisinos); Universidade do Vale do Taquari (Univates); Universidade Católica de Pelotas (UCPel); Universidade de Passo Fundo (UPF); Universidade da Região da Campanha (Urcamp); e Universidade Regional Integrada do Alto Uruguai e das Missões (URI).

El COMUNG está constituido por ese conjunto de instituciones con el objetivo de integrar y fortalecer individualmente a las instituciones de enseñanza superior comunitarias de Rio 


\section{Journal of the Academy $|83|$}

Grande do Sul (COMUNG, 2020a). (Palmeiras y Grzybovski, 2016) explican que el COMUNG actúa conjuntamente, estableciendo políticas y convenios de manera a incentivar la comunidad académica en lo que se refiere a la formación, promoción de actividades culturales, de innovación, emprendedurismo, etc.

En este camino, destacamos la importancia de la gestión sostenible, la cual ha sufrido cambios, ya que el concepto y la forma de organizar y promover la educación han ido cambiando y, por tanto, requiriendo nuevas estrategias (Tofik, 2013). Según Colombo (2004), la gestión académica es responsable de aportar ventajas a los modelos de interdisciplinaridad y transversalidad, además de formar a los docentes en una perspectiva proactiva a las necesidades del mercado laboral. También sobre ese tema, Oliveira y Della Giustina (2018) explican que es deber de la gestión de la institución posibilitar que los estudiantes accedan y construyan conocimientos de manera democrática. En esa perspectiva, la gestión académica consiste en una articulación de acciones orientadas a la política educativa democrática, poniendo el aprendizaje y la enseñanza en el centro de las decisiones de los directivos, buscando el comprometimiento de los docentes con las políticas institucionales.

Otro término que es necesario definir en este marco teórico debido a su relevancia, es la coopetición. En un escenario de competencia entre las muchas instituciones de educación superior (IES), entendemos que, si bien compiten por la captación y permanencia de los estudiantes, tienen intereses similares. Así, además de competir, las IES pueden y deben cooperar simultáneamente en la búsqueda de objetivos comunes, lo que se conceptualiza como coopetición (Czachon y Mucha-kuś, 2014). De acuerdo con los autores, la coopetición se diferencia de la cooperación y de la competición, por ser un proceso que ocurre simultáneamente. Según Das Neves et al. (2011) la cooperación es una nueva forma de pensar sobre los negocios, o incluso una estrategia que se puede desarrollar.

En la concepción de Dal-Soto y Monticelli (2017), lo ideal es que exista una coopetición, es decir, una relación estratégica entre las empresas o instituciones, en la que, a la vez que compiten, también cooperan de forma simultánea. En el mismo sentido, Santos y Mugnaini (2019) se refieren a este término como una estrategia de relación en la que hay, simultáneamente, competencia y cooperación. 


\section{Journal of the Academy $\mid 84$ |}

Bengtsson et al. (2014) afirman que la coopetición, es decir, el movimiento simultáneo de cooperar y de competir entre empresas se ha incrementado, convirtiéndose en un punto relevante en la agenda de muchas organizaciones. En el mismo sentido camina la comprensión de Dal-Soto y Monticelli (2017), los que, además, añaden que la coopetición brinda una situación de interdependencia entre las empresas, con parcial convergencia de intereses y de objetivos. Así, entendemos que la cooperación acompañada de competencia, reduce gastos y aporta nuevos conocimientos, y asimismo recursos complementarios (Bobera et al., 2017).

Como explican Santos y Mugnaini (2019), la coopetición es una estrategia comúnmente encontrada en los sectores empresariales, pero los autores creen en los resultados positivos cuando se aplica al área educativa. En este sentido, se puede apreciar que las Instituciones Comunitarias de Educación Superior del COMUNG tienen ese vínculo, pues compiten por los estudiantes, pero también cooperan en la divulgación de su relevancia social, en el incremento del incentivo económico de parte del gobierno, entre otros.

Así, se puede ubicar la coopetición en el modelo teórico de gobernanza universitaria presentado por Fossatti et al. (2017), es decir, una forma de conducir una IES a que alcance: a) Fortalecer el núcleo de dirección, estimulando la participación de todos los involucrados, b) Integrar la organización, con miras a mejorar la comunicación interna y externa, c) Profesionalizar la administración, alejando la politización, el amiguismo y el clientelismo, d) Estimular el corazón del mundo universitario por medio del incremento del perfil personal académico, y e) Desarrollar una cultura emprendedora. Habiendo abordado los modelos de gobierno Burocrático (formal y preciso), Colegial (compartido), de Partes interesadas (abierto), Emprendedor (de liderazgo), de Anarquía organizada (no formal) y Político (negociable), los autores llegaron a la conclusión que, especialmente en América Latina, no es posible que tenga un gobierno universitario basado en un modelo único, sino en comunión de varios de ellos. Sin embargo, hay que garantizar dos importantes logros: el crecimiento del número de alumnos a través de una enseñanza de calidad, y el desarrollo de la comunidad en la está insertada la universidad.

De esa manera, se puede percibir que la coopeticón, a la vez que crea una sinergia entre las empresas que cooperan y al mismo tiempo compiten, surge como un modelo de gobernanza que se apoya en la fuerza conjunta de instituciones que buscan objetivos comunes y que se fortalecen su segmento ante al mercado. Esa fortaleza se hace percibir en la sociedad, formando 


\section{Journal of the Academy $|85|$}

lo que Cezarino y Campomar (2006) referirán como clusters, que significan la formación de redes de instituciones, lo que, según los autores, es imprescindible en un mundo globalizado. Así, los clusters son una concentración geográfica de conjuntos productivos (en el caso del COMUNG, limitándose al estado brasileño del Rio Grande do Sul), de sectores o actividades económicas que agreguen conocimiento, capital físico o capital humano (universidades comunitarias), genéricamente denominados en la literatura de agrupamientos, y aquí generando un fenómeno llamado de coopetición.

\section{METODOLOGÍA}

El objetivo de este artículo consiste en sistematizar las experiencias de coopetición entre las IES del COMUNG por medio de la participación en actividades promotoras de gestión sustentable. De acuerdo con Prodanov (2013) hay un vínculo irrompible entre el mundo subjetivo y el mundo objetivo, que no puede traducir en números. Desde esta perspectiva, el abordaje de la investigación el cualitativo, definido por Bardin (2011) como aquel que se basa en componentes subjetivos y que no rechaza cualquier dato cuantitativo, pues comprende que ellos podrán ser utilizados como forma de fortalecer el corpus investigativo.

Para el alcance de nuestro objetivo, realizamos un estudio de caso. Según aclara Yin (2015), este tipo de estrategia investigativa está indicada para la búsqueda de respuestas a las preguntas cómo y por qué, cuando no se tiene total control sobre los ocurridos, y aún cuando hay una proximidad del objeto (o de los objetos) de estudio a la contemporaneidad y a la vida real. Así, nuestra unidad de análisis está conformada por el Consorcio de Universidades Comunitarias de Rio Grande do Sul, compuesto por 14 IES.

Para ello, realizamos una revisión de la literatura que, según Moresi (2003) consiste en el marco teórico para el abordaje de un tema y problema investigativo. Para la recolección de datos también se realizó, como investigadores y gestores involucrados en esta narrativa, observaciones de las acciones de coopetición entre las instituciones del COMUNG, registradas en un diario de campo. Por lo tanto, no realizamos recopilaciones externas, sino que recopilamos datos, experiencias e informes de los autores de este estudio. 


\section{Journal of the Academy $|86|$}

Para nuestra revisión, se utilizó los descriptores coopetición, gestión y educación superior, en las bases de datos Periódicos Capes ${ }^{5}$, Scielo y EBSCO. Luego de la primera búsqueda, que resultó en un total de 110 materiales, se leyeron los resúmenes con el fin de verificar la relevancia de los temas, resultando en 30 materiales seleccionados. Hecho eso, luego de leer los textos en su totalidad, se publicaron seis artículos, cuatro libros, dos disertaciones y dos artículos completos en los anales de eventos, como se muestra en la figura siguiente.

\section{Figura 1}

\section{Revisión de literatura}

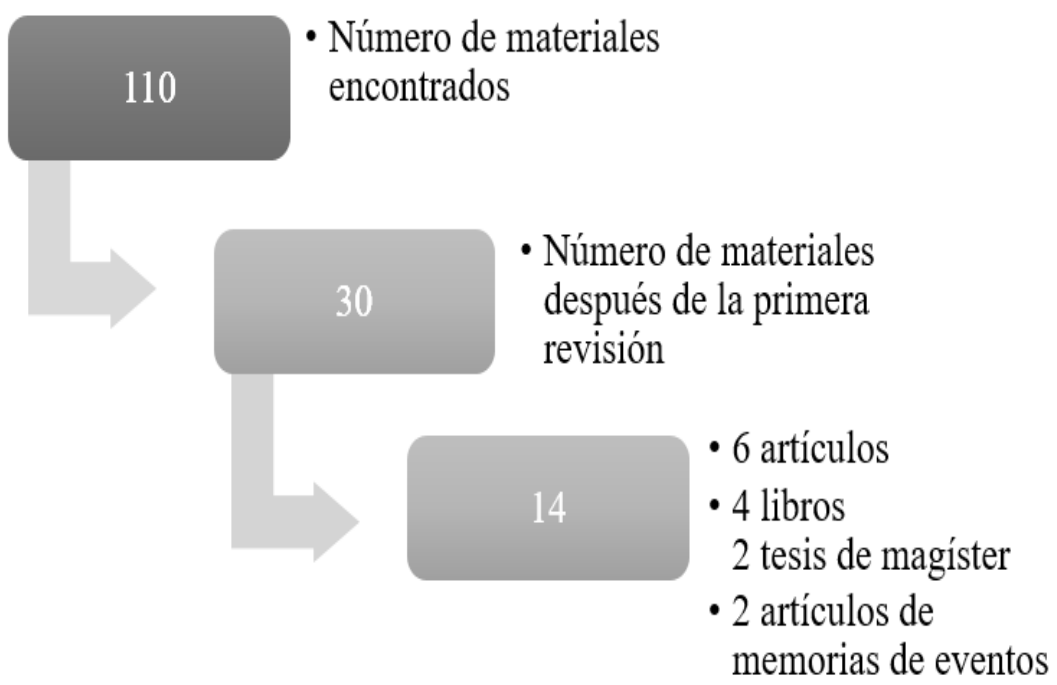

Fuente: Elaboración propia (2020).

En cuanto al análisis de los hallazgos, nos apoyamos en la técnica de Análisis de contenido de Bardin (2011). Según la autora, podemos entender este método como un conjunto de técnicas y procedimientos con el fin de comprender el contenido de determinados mensajes, considerando los significados, es decir, el contenido y su contexto.

\section{RESULTADOS}

La coopetición en nivel del COMUNG puede ser observada en varias acciones. Desde la constitución de una red de formación de docentes, con carácter interinstitucional, que organizó

\footnotetext{
${ }^{5}$ Sitio de la Coordinación para Perfeccionamiento del Personal de Educación Superior (Capes), órgano del Ministerio de Educación brasileño, responsable de la expansión y consolidación de cursos de posgrado stricto sensu (maestría y doctorado) en todos los estados brasileños. Disponible en: https://catalogodeteses.capes.gov.br/catalogo-teses/\#!/ Acceso el 12.12.2020.
} 


\section{Journal of the Academy | 87|}

y ofreció, desde principios de 2019 hasta diciembre de 2020, una especialización en docencia de Educación Superior con y para docentes del Consorcio, hasta la constitución de un comité de gestión, que se encarga de pensar y articular estas acciones en red. Como explican Dal-Soto y Monticelli (2017), la coopetición implica simultáneamente estrategias de cooperación y competición entre empresas en diferentes áreas y bajo diferentes niveles de interacción. Un concepto similar trae Duarte (2019), afirmando que, por medio de la coopetición se crea valor entre las empresas, de forma que se armonizan intereses distintos hacia un objetivo común, creando oportunidades y ventajas competitivas a las instituciones involucradas, posibilitando la eliminación de obstáculos y amenazas externas.

Otro ejemplo que puede citado fue el Congreso de prácticas docentes Comung Conversa - do presencial ao virtual: experiências docentes no ensino superior em tempos de pandemia (COMUNG, 2020b), evento totalmente online. Más de 340 profesores de COMUNG han participado en el intercambio de experiencias durante los tres días del evento.

Además, durante 2019 hubo un movimiento de renovación curricular de todas las IES del COMUNG como articulación en red. Las acciones pensadas y realizadas por el COMUNG atraen la atención de varios investigadores, especialmente el prof. Dr. Marcos Tarciso Masetto $^{6}$, que ha participado con su grupo de investigación en algunos encuentros institucionales promovidos por el Consorcio. Según Masetto (2014), el proceso de innovación universitaria requiere trabajar juntos, porque innovar significa hacer lo nuevo y hacerlo juntos.

Sin embargo, para este estudio nos centramos en dos categorías encontradas a partir del examen de los datos de la investigación, que se justifican por el contexto mundial actual de pandemia, aislamiento social y educación remota en todas las IES del COMUNG, en el primer semestre del año de 2020: a) La coopetición como estrategia para fortalecer las instituciones educativas en tiempos de crisis junto de agencias gubernamentales; b) La cooperación como espacio de fortalecimiento de las universidades para la gestión sostenible.

\section{El contexto de la educación remota}

\footnotetext{
${ }^{6}$ El profesor Marcos Masetto es magíster y doctor en Psicología de la Educación (PUCSP) y Libre Docente de Didáctica (USP). Lleva 55 años enseñando en la Educación Superior.
} 


\section{Journal of the Academy $|88|$}

En enero de 2020, el COVID-19 se consideró una epidemia y, el 11 de marzo del mismo año, la Organización Mundial de la Salud definió una pandemia, ante la alta tasa de transmisión del virus. Ante esta situación, en Brasil, siguiendo las tendencias globales, varios estados y municipios han promulgado medidas de aislamiento social como una forma de detener la evolución de la enfermedad.

En el segmento educativo, se buscaron alternativas para continuar las actividades desarrolladas en escuelas y universidades. Un país con dimensiones continentales, y entre los más grandes en desigualdades sociales, Brasil tiene varios escenarios en la lucha contra el coronavirus en lo que respecta a la continuidad de la educación por medios remotos. En todo el país, los sistemas educativos comunitarios, tanto privados como estatales, siguiendo los lineamientos del Ministerio de Educación, han elaborado sus planes de contingencia para, en muy poco tiempo, sin condiciones para grandes discusiones, tomar decisiones sobre la interrupción total, parcial o continuidad de las actividades educativas por sistema remoto.

El mapa brasileño registró diferentes escenarios, como, por ejemplo, el cierre temporal de escuelas periféricas, independientemente de que sean estatales, privadas o comunitarias, la continuidad a través de la educación remota, a ejemplo de escuelas de clase media y alta. En la educación superior, las universidades privadas y comunitarias, en su mayor parte, se dirigieron a la educación a distancia, como fue el caso de las IES del COMUNG. Las universidades estatales, en cambio, debido a una cultura centrada en la educación presencial, encontraron mayores dificultades para continuar los estudios no presenciales, muchas de ellas suspendiendo temporalmente las clases en cualquier modalidad. Según el sitio de noticias G1, de las 69 IES estatales, solo seis continuaron con educación remota en el período (Paixão, 2020).

En cuanto a las IES del COMUNG, considerando la trayectoria de servicio a la comunidad en sus proyectos sociales, la identidad de profundo arraigo comunitario y la formación en educación digital, el camino elegido recayó en la educación remota en el sistema sincrónico. Esta estrategia mantuvo el dinamismo de sus instituciones respetando las limitaciones impuestas por COVID-19. Como destacan Fossatti et al. (2019), hay que conectar la enseñanza a las necesidades locales. La investigación es una competencia intelectual, pero tiene sentido cuando se interrelaciona con el alcance comunitario. 


\section{Journal of the Academy | 89 |}

En este período de grandes desafíos financieros, económicos, académicos, sociales y de escasa aproximación social para esta generación, el COMUNG, a través de recursos virtuales, se organizó rápidamente con estrategias de sostenibilidad. Entre ellos, los dos mencionados anteriormente se destacan y ganan espacio de discusión a continuación. Como explica Fava (2016) los tiempos de crisis demandan menos presupuesto y más creatividad. Hay que capacitar a los equipos para que desarrollen diferenciales creativos es una forma de integrar a los educadores e investigar estrategias de afrontamiento efectivas dentro de la empresa.

\section{La coopetición como estrategia de fortalecimiento de instituciones educacionales en tiempos de crisis junto a órganos gubernamentales}

En tiempos de crisis, la premisa de que tendremos más posibilidades de sobrevivir si permanecemos juntos, que también se considera coopetición en sentido amplio, gana destaque. Este pensamiento estuvo presente en el COMUNG, mostrándose en varias acciones. Encabezada por las reuniones sistemáticas de los rectores, cada dos semanas, la agenda giró en torno a la toma conjunta de decisiones para el fortalecimiento institucional en este momento de pandemia con el gobierno estatal y los respectivos gobiernos municipales. En este sentido, Fava (2016) anuncia que es necesario, al reconsiderar las prioridades, reformular también las mentalidades. Si bien no considere una tarea sencilla, la creatividad y la innovación son ahora sintomáticas para la supervivencia y para la longevidad de cualquier institución educativa.

La decisión recayó en el diálogo entre el COMUNG y la Secretaría de Desarrollo y Tecnología del Estado de Rio Grande do Sul. Este canal de diálogo condujo al establecimiento de una agenda común, guiada por un trabajo colaborativo centrado en la investigación para combatir y prevenir el COVID -19. Además de la colaboración como entidad, las IES de forma individual también compiten entre sí para mostrar a la sociedad y a los medios de comunicación acciones que las legitimen en su carácter comunitario. Entonces, de acuerdo con Fava (2016), cada idea se mejora constantemente, desarrollándose nuevas oportunidades.

El mismo movimiento ocurre en la producción de materiales de protección en la lucha contra el coronavirus. Al mismo tiempo que las IES socializan sus proyectos, se esfuerzan por dar visibilidad institucional en su área de actuación, con acciones como donaciones a hospitales y puestos de salud. Este comportamiento se muestra a diario en el diálogo entre el COMUNG y los gobiernos, con resultados optimistas en el cumplimiento de los protocolos establecidos y 


\section{Journal of the Academy $|90|$}

acciones efectivas, así como de cada IES con sus municipios e instituciones asociadas. También cooperan y compiten simultáneamente para atender a los intereses de los estudiantes en situación de vulnerabilidad social, en la demanda de acceso a internet y en nuevas formas de financiamiento estudiantil. Esto significa que la institución educativa contemporánea, según Fossatti et al. (2019), ya no puede funcionar como un estante lleno de cajones, en los que cada uno actúa aisladamente. Se trata, sobre todo, de un trabajo cooperativo, ya sea con el público interno de la IES, o con el público externo, pero también y, principalmente, con las instituciones asociadas, como se ha visto con Fossatti et al. (2017), cuando los autores desarrollan el concepto de gobernanza universitaria, pues aquí se trata de llamar la atención para el mejoramiento de la calidad de la educación para incrementar en número de alumnos. Además, acciones como esas no caminan lejos del concepto de desarrollo de la comunidad en la se insertan las IES del COMUNG.

Junto a los organismos de Salud, la comunidad también realizó un doble movimiento: en cadena e individualmente, abrieron las puertas de sus laboratorios, facilitando la fabricación de alcohol en gel, la confección de protectores faciales; aplicación de pruebas para detectar COVID-19; proyectos para el desarrollo de vacunas y respiradores artificiales, entre otros. En vista de su naturaleza comunitaria, las 14 IES del Consorcio están comprometidas, con sus equipos de salud, a servir a su comunidad. Como proclaman Schafer y Spengler (2019) a las universidades comunitarias cabe no solo la oferta educativa, sino también cumplir el rol de social como política de inclusión. Esto significa que los proyectos, las becas que se ofrecen, las prácticas, todo el trabajo que involucra el trípode de enseñanza, investigación y extensión debe beneficiar a la comunidad en la que están insertadas esas instituciones.

Este movimiento colectivo fortaleció a las IES del COMUNG en el momento de aclarar a los parlamentarios en Brasilia (capital nacional), que habían presentado propuestas de Proyecto de Ley solicitando la reducción de las cuotas mensuales, que esta práctica sería inconstitucional frente a los compromisos asumidos, el aumento del gasto en tecnologías de la información para dar calidad a clases remotas en el modelo síncrono, entre otros. Así, los rectores, a través de sus comisiones y asociaciones constituidas, como la Asociación Brasileña de Instituciones Comunitarias de Educación Superior (ABRUC), la Asociación Nacional de Educación Católica (ANEC) y el Consejo de Rectores de Universidades Brasileñas (CRUB) pudieron ser escuchados a través de cartas y documentos comunes e individuales firmados por estas entidades. Aún en este sentido, continúan buscando estrategias colectivas y aisladas, 


\section{Journal of the Academy | $91 \mid$}

financiamiento para estudiantes que se encontraron en una situación financiera difícil con el advenimiento de la pandemia y el consecuente aislamiento social. Se percibe aquí claramente como un cluster (Cezarino y Campomar, 2006), por medio de estrategias de coopetición muestra su fuerza junto a otros segmentos públicos y privados, posibilitando la eliminación de obstáculos y amenazas externas (Duarte, 2019).

También es importante destacar la participación efectiva del COMUNG en la elaboración de decretos estatales y municipales durante el período pandémico. Las contribuciones efectivas fueron consideradas en los protocolos que conducen al cruce del período. A continuación, presentamos el resumen de estas acciones que muestran el resultado de la coopetición a nivel estatal y municipal, una realidad construida por las IES del COMUNG como una forma de legitimar su identidad ante sus respectivas comunidades.

\section{Tabla 2}

Síntesis de las acciones realizadas por las IES del COMUNG sobre el COVID-19

\section{Acciones}

Orientación sobre cuidado de la salud física, alimentación saludable, salud psicológica y sanitaria

Equipo de protección, como protectores faciales

Diagnóstico del COVID-19 y Vacunas de la Gripe

Producción y distribución gratis de material de higiene y limpieza, como alcohol en gel, jabones, alcohol líquido, alimentos y ropa

Atención online

Investigaciones sobre coronavirus

Atención al comercio y pequeños emprendedores

Cursos, webinars, lives, conferencias y actividades online gratis

Consejos y estrategias para rutina en casa y/o home office

Fuente: Elaborado por los autores a partir de los datos de la investigación.

\section{La coopetición como espacio de fortalecimiento entre las universidades para una gestión sostenible}

El movimiento de mirar hacia afuera, en asociación con los gobiernos, ocurre en paralelo con el movimiento de mirar hacia adentro de cada IES y el conjunto de estas instituciones. Es decir, 


\section{Journal of the Academy $|92|$}

la cooperación también ocurre de manos dadas con la competencia, en el momento que cada uno necesita garantizar su viabilidad, y en coopetición, equilibrando intereses de cada IES e intereses colectivos. Como explica Monticelli (2015), acciones de coopetición empiezan a reemplazar un paradigma puramente competitivo, generando nuevas formas de gobernanza intraorganizacional y que permiten la ampliación de alternativas de agrupamiento de las empresas.

Si bien cada una de las IES del COMUNG tiene sus especificidades y características con respecto a la gobernanza universitaria, el trabajo en red del Consorcio también permite la búsqueda de decisiones ágiles estratégicas, tácticas y operativas para enfrentar los desafíos que se presentan. En cuestión de una semana, casi 190 mil académicos de las 14 IES del COMUNG (COMUNG, 2020a) pasaron a tener clases a distancia, lo que exigió un esfuerzo concertado entre las instituciones, sus líderes, docentes y equipos en el área de tecnologías y recursos digitales. El compartir entre las IES las fortaleció a todas, tanto a nivel individual como en la representatividad del grupo.

Además, la definición de estrategias de preparación para la realidad que se enfrentará después del aislamiento social también ha sido tema de discusiones en red con líderes en el contexto del COMUNG. Una vez más, la relación de intercambio de información y experiencia ha beneficiado a todas las universidades involucradas. En este sentido, Bittar (2001) explica que las IES comunitarias poseen un fuerte compromiso con el trabajo académico de excelencia, con la resolución de problemas de los marginados, con la búsqueda de ideales de ciudadanía, solidaridad y democracia. Esas características hacen que las universidades comunitarias en Brasil sean cada vez más conocidas y respetadas por su actuación en la esfera académica, científica, pública y social.

Dado el contexto, en cuestión de dos semanas, el COMUNG, que antes estaba prácticamente limitado a los rectores, ahora comienza a operar con varios grupos. Entre ellos destacamos: Grupo de Pro-Rectores Académicos para la socialización de buenas prácticas en educación a distancia; estrategias para la formación de docentes en línea y envío de sugerencias para protocolos de gobierno estatal y municipal. Entre las iniciativas puestas en común se encuentran la asistencia sanitaria en el seguimiento de sus proyectos sociales; asistencia psicológica en línea a estudiantes; profesionales de la salud y comunidad en general; participación efectiva en las propuestas de decretos y protocolos estatales y municipales. Estas 


\section{Journal of the Academy $\mid 93$ |}

estrategias muestran cooperación con los gobiernos y con la sociedad al mismo tiempo que provocan una competencia sutil al mostrarle a la sociedad lo mejor que está haciendo cada IES. Esta tarea está muy bien realizada por los equipos de comunicación y marketing. Como dice Fava (2016), los equipos que se superan en hacer más con menos recursos sobrepasan las expectativas con actitud, espíritu creativo e innovador. De esa forma, se muestran capaces desarrolla productos y servicios más atractivos y eficientes.

El grupo de Pro-Rectores Administrativos se forma para dialogar con los diferentes sindicatos con el fin de fortalecerse en todas las decisiones y negociaciones, así como para reforzar el intercambio de prácticas de buen gobierno. También se reunieron para representar el COMUNG a nivel federal en la discusión y presentación de propuestas de financiamiento estudiantil e institucional con parlamentarios y ministerios. En este escenario, se percibe muy claramente el concepto de coopetición que refleja Monticelli (2015) cuando explica que en la relación de coopetición, los agentes cooperan en determinadas actividades y, simultáneamente, compiten en otras.

Asimismo, los responsables de Movilidad Académica e Internacionalización buscaron soluciones para sus respectivas IES de manera conjunta en cuanto a la movilidad de estudiantes y docentes en Brasil y en el exterior. Fruto de esta nueva forma de hacer educación, la internacionalización en casa ha ido ganando varias propuestas de implementación ante las dificultades con los desplazamientos geográficos.

Finalmente, los Asesores Jurídicos también han utilizado la fuerza personal y colectiva para comprender y aplicar la nueva legislación durante la pandemia, así como en la consultoría mutua para la toma de decisiones. Ante las propuestas de un Proyecto de Ley que visaba reducir las tasas de matrícula en línea, los departamentos legales sensibilizaron a la prensa y a los parlamentarios sobre el error de esa posible decisión y defendieron la negociación personal de acuerdo con la realidad de cada estudiante. La tabla 3 resume las principales acciones llevadas a cabo durante el período pandémico. Llamamos la atención sobre muchas iniciativas en tan poco tiempo, que se limita a dos meses, desde el inicio de la cuarentena hasta la fecha de conclusión de la escritura de este artículo.

\section{Tabla 3}

Acciones COMUNG para fortalecer la gestión sostenible en tiempos de coronavirus 


\begin{tabular}{ll}
\hline Rectores & $\begin{array}{l}\text { Reuniones sistemáticas de las Rectorías. } \\
\text { Representatividad ante el Gobierno del Estado y Comités Municipales. }\end{array}$ \\
$\begin{array}{l}\text { Pro-Rectores } \\
\text { Académicos }\end{array}$ & $\begin{array}{l}\text { Intercambio de buenas prácticas en educación remota. } \\
\text { Participación en la elaboración de protocolos gubernamentales. } \\
\text { Elaboración conjunta de guías para protocolos de retorno post-pandemia. }\end{array}$ \\
$\begin{array}{l}\text { Pró-Rectores } \\
\text { Administrativos }\end{array}$ & $\begin{array}{l}\text { Negociaciones sindicales. } \\
\text { Búsqueda conjunta de financiación. } \\
\text { Construcción conjunta de lineamientos para la sostenibilidad financiera de } \\
\text { las instituciones. }\end{array}$ \\
$\begin{array}{l}\text { Responsables de } \\
\text { Movilidad }\end{array}$ & $\begin{array}{l}\text { Acompañamiento a alumnos en el exterior y a extranjeros en nuestras IES. } \\
\text { Refuerzo de prácticas para la Internacionalización en Casa. }\end{array}$ \\
Internacionalización & \\
Consejos Jurídicos & $\begin{array}{l}\text { Estudio conjunto de la nueva legislación resultante de la pandemia } \\
\text { Documentos firmados por las IES con el gobierno y parlamentarios a nivel } \\
\text { estatal y federal. }\end{array}$
\end{tabular}

Fuente: elaborado por los autores, a partir de datos de la investigación (2020).

Como vimos, esta interacción entre empresas adquiere la connotación de un juego de estrategia en el que el posicionamiento de un agente influye en la toma de decisiones del otro (Monticelli, 2015). Además, a través de este relato de experiencia, nos damos cuenta de que esta interacción termina influyendo en la visibilidad de las instituciones involucradas con los organismos gubernamentales, así como en el fortalecimiento de la gestión sostenible, como es el caso del escenario presentado.

\section{CONCLUSIONES}

El texto registra la presentación de la experiencia de coopetición vivida por las IES del COMUNG en un corto periodo durante la pandemia, ante a la problemática de la investigación que se ha planteado: ¿qué acciones han llevado a cabo las IES comunitarias del COMUNG para promover la gestión sostenible y que se pueden caracterizar como experiencias de coopetición? Aunque pueda ser considerado un período muy pequeño, en el criterio cronológico, el periodo se comporta intensamente en el componente existencial, así como en la capacidad de reorganización y toma de decisiones colectivas. 


\section{Journal of the Academy $\mid 95$ |}

Los resultados registran el fortalecimiento de las 14 instituciones del COMUNG con agencias gubernamentales, en forma de cluster, empoderando las voces de la educación superior comunitaria, en un modelo de gobernanza universitaria orientado a acciones conjuntas, aquí llamadas coopetición. También muestran el alto grado de responsabilidad social hacia sus comunidades en la satisfacción de necesidades básicas, no limitadas a cuestiones de salud. Asimismo, la coopetición forja el empoderamiento interno de cada IES para enfrentar sus situaciones, límites y desafíos, ya que funciona con la lógica de cooperación externa ante la comunidad y el gobierno, de cooperación entre otras IES en la dialéctica con estrategias y competencia para garantizar su lugar en el mercado ante sus players.

Como limitaciones del estudio, se menciona el corto período al que se refiere el análisis, lo que plantea trabajos futuros, con datos empíricos, que pueden confirmar las inferencias de esta investigación. Cuando volvamos a las actividades cara a cara, después de la pandemia y después de la vacunación masiva, probablemente seremos capaces de mirar hacia atrás y hacer nuevas consideraciones con respecto a este período de aprendizaje y desafíos.

Se concluye por la coopetición como una estrategia asertiva y potencializadora al empoderamiento del COMUNG para enfrentar sus desafíos en tiempos de COVID-19. Muestra una posibilidad de gobernanza universitaria abierta y prometedora para las instituciones del Consorcio, a fin de fortalecer su ventaja competitiva. Se puede inferir que la cooperación también puede beneficiar a otras IES, de carácter estatal o privado, ya que potencia el capital humano, intelectual, los recursos materiales y el poder político en la toma de decisiones.

El texto avanza el conocimiento al legitimar la coopetición como categoría posible para afrontar periodos de grandes retos, como el que estamos viviendo. Sugerimos y pretendemos continuar con el estudio longitudinal para evaluar la efectividad e intensidad de la coopetición también en el periodo post-pandemia 


\section{REFERENCIAS BIBLIOGRÁFICAS}

Bardin, L. (2011). Análise de conteúdo. Edições 70.

Bengtsson, M., Kock, S., Lundgren-Henriksson, EL y Näsholm, MH (2016). Pesquisa de coopetição na teoria e na prática: cultivando novos domínios teóricos, empíricos e metodológicos. Gestão de Marketing Industrial, 57, 4-11.

Bittar, M. (2001). O ensino superior privado no Brasil e a formação do segmento das universidades comunitárias. Revista da Avaliação da Educação Superior, Campinas, 6(2), $33-42$.

Bobera, D.; Leković, B. y Strugar-jelača, M. (2017). Coopetition as an external source for driving innovation: Research findings from AP Vojvodina. Ekonomski pogledi, 19(1), 116. https://scindeks-clanci.ceon.rs/data/pdf/1450-7951/2017/1450-79511701001B.pdf

Brasilia, DF. (2013). Lei $n^{\circ} 12.881$, de 12 de novembro de 2013. Lei da definição, qualificação, prerrogativas e finalidades das instituições comunitárias de educação superior. Brasília, DF.

Cezarino, L. O. y Campomar, M. C. (2006). Vantagem competitiva para micro, pequenas e médias empresas: clusters e APLs Competitive advantage to small and medium business: cluster and LPAs. Revista Economia \& Gestão, 6(12). http://periodicos.pucminas.br/index.php/economiaegestao/article/download/37/32

Colombo, S. S. (2004). Gestão Universitária: Os Caminhos para a Excelência. Penso.

Consórcio das Universidades Comunitárias Gaúchas. (2020a). Sobre o COMUNG. Consórcio das Universidades Comunitárias Gaúchas. https://COMUNG.org.br/sobre.

Consórcio das Universidades Comunitárias Gaúchas. (2020b) Seminário Comung Conversa do presencial ao virtual: experiências docentes no ensino superior em tempos de pandemia. Memorias. https://www.univates.br/editora-univates/publicacao/337

Czachon, W. y Mucha-kús, K. (2014). Coopetition research landscape-a systematic literature review 1997-2010. Journal of Economics \& Management, 17 (1), 122-150. http://yadda.icm.edu.pl/yadda/element/bwmeta1.element.desklight-e54a1134-12c2-4b29918e-b1c5c71f967d.

Dal-soto, F. y Monticelli, J. (2017). Coopetition strategies in the Brazilian higher education. Revista de Administração de Empresas, 57 (1), 65-78. https://www.scielo.br/pdf/rae/v57n1/0034-7590-rae-57-01-0065.pdf

Das Neves, M. P. S., Hansen, P. B., Diehl y C. A. y Becker, G. V. (2011). Análise do processo de coopetição em redes horizontais de pequenas e médias empresas do Rio Grande do Sul. Base (UNISINOS), 8 (3), 243-260.

Duarte, M. J. L. (2019). Colaboração em inovação e concorrência. Dissertação de mestrado (Economia Industrial e da Empresa) - Universidade do Minho. Repositorium SDUM. 


\section{Journal of the Academy | $97 \mid$}

https://repositorium.sdum.uminho.pt/bitstream/1822/60514/1/TESE\%2BFINAL\%2B\%2BMaria\%2BDuarte.pdf.

Fava, R. (2016). Educação para o século XXI: a era do indivíduo digital. Editora Saraiva.

Fossatti, P., Ganga, F. y Jung, H. S. (2017). Reflexiones en torno a la gobernanza universitaria: Una mirada desde Latinoamérica. Revista Espacios, 38(35). https://www.revistaespacios.com/a17v38n35/17383514.html

Fossatti, P.; Güths, H. y Jung, H. S.. (2019). Gestão educacional: contingências da contemporaneidade. In: Moll, J.; Fensterseifer, D., Vázquez, J. M. Memórias do X Sincol: Simpósio Nacional de Educação. Frederico Westphalen: Editora da URI, p. 36-52.

Masetto, M. (2014). Docência na universidade. Papirus Editora.

Monticelli, J. M. (2015). Competição, cooperação e coopetição: simetrias e discrepâncias na indústria vitivinícola do RS. Revista de Administração Unimep, 13 (2), 1-25.

Moresi, E. (2003). Metodologia da pesquisa. Universidade Católica de Brasília.

Oliveira, W. A. y Della Giustina, F. P. (2018). Gestão educacional: relação docente/discente no ensino superior. Revista de Enfermagem da FACIPLAC, 1 (1). http://revista.faciplac.edu.br/index.php/REFACI/article/view/503

Paixão, A. (2020) Só 6 das 69 universidades federais adotaram ensino a distância após paralisação por causa da Covid-19. G1 Globo. https://g1.globo.com/educacao/noticia/2020/05/14/so-6-das-69-universidades-federaisadotaram-ensino-a-distancia-apos-paralisacao-por-causa-da-covid-19.ghtml

Palmeiras, J. de B. y Grzybovski, D. (2016). Perfil do gestor e desempenho econômicofinanceiro das instituições de ensino superior: o caso das comunitárias brasileiras participantes do COMUNG. In: Coloquio Internacional de Gestión Universitaria, XVI, 2016, Arequipa, Perú.

Prodanov, C. C. (2013). Metodologia do trabalho científico: métodos e técnicas da pesquisa e do trabalho acadêmico. 2. ed. Feevale.

Quadros Da Silva, L. de. (2019). Novas metodologias na gestão acadêmica universitária: Um estudo de caso na unilasalle canoas. Dissertação (Mestrado em Educação) - Universidade La Salle. Repositorio Universidad Lasalle. http://repositorio.unilasalle.edu.br/handle/11690/1192?mode=full

Santos, S. M. Dos y Mugnaini, R. (2019). As Universidades Estaduais Paulistas e as Ciências Agrárias: Oportunidades de Coopetição. MARCOVITCH, J. Repensar a Universidade: Impactos para a Sociedade. Com-Arte, Fapesp. p. 159-194.

Schafer, R. P. y Spengler, F. M. (2019). O papel social das universidades comunitárias enquanto terceiro no tratamento dos conflitos. Revista Direito \& Paz, 2 (41), 91-107. http://www.revista.unisal.br/lo/index.php/direitoepaz/article/view/1140/495

Schmidt, J. P. (2018). Universidades comunitárias e terceiro setor [recurso eletrônico]: fundamentos comunitaristas da cooperação em políticas públicas. 1. ed. Santa Cruz do Sul: 


\section{Journal of the Academy $|98|$}

EDUNISC.

https://repositorio.unisc.br/jspui/bitstream/11624/2731/1/Universidades\%20comunit\%c3 \%a1rias\%20e\%20terceiro\%20setor.pdf

Tofik, D. S. (2013). A gestão acadêmica nas instituições de ensino superior. In: Colombo, S. S. Gestão Universitária: Os Caminhos para a Excelência. Penso.

Yin, R. K. (2015). Estudo de caso: Planejamento e métodos. $5^{\circ}$ ed. Bookman. 